\title{
IMUNOSSUPRESSÃO E PROTEINÚRIA
}

\author{
Immunosuppression and proteinuria
}

\author{
Andreia Fabiana do Vale Franco ', Rodrigo José Ramalho ', Irene de Lourdes Noronha',2
}

\begin{abstract}
RESUMO
A proteinúria é um importante marcador de lesão renal, não apenas na doença renal crônica como também em transplante renal. Nos pacientes transplantados renais a proteinúria pode ter diversas causas, tais como: recidiva da doença de base, nefropatia crônica do enxerto, glomerulopatia do transplante e glomerulopatia de novo. As drogas imunossupressoras utilizadas em transplante interferem de diversas formas na excreção urinária de proteínas. Os inibidores de calcineurina apresentam importante efeito antiproteinúrico, sendo atualmente utilizados no tratamento de glomerulopatias primárias. Efeito antiproteinúrico também foi descrito com o uso de micofenolato. No entanto, alguns estudos têm descrito o aparecimento e/ou aumento de proteinúria relacionado ao uso de inibidores do sinal de proliferação (PSIs) com níveis variados, podendo alcançar níveis nefróticos. Existem evidências clínicas de que a proteinúria responde ao uso de inibidores da enzima de conversão ou bloqueadores do receptor de angiotensina. Nesse contexto, o estudo do efeito de PSIs sobre as proteínas da fenda diafragmática dos podócitos, como a nefrina, podocina e sinaptopodina (entre outras) e também sobre o fator de crescimento vascular endotelial (VEGF), é importante para esclarecer o aparecimento da proteinúria em pacientes transplantados. A presente revisão aborda os aspectos acima mencionados.
\end{abstract}

Descritores: Transplante de Rim, Proteinúria, Imunossupressores, Podócitos, Fator de crescimento do endotélio vascular.

Instituições:

${ }^{1}$ LIM 29 - Laboratório de Nefrologia Celular, Genética e Molecular - Faculdade de Medicina Universidade de São Paulo - São Paulo / SP - Brasil

${ }^{2}$ Clínica de Nefrologia do Hospital Beneficência Portuguesa - São Paulo / SP - Brasil

Correspondência:

Prof $^{\text {a }}$ Dr $^{\mathrm{a}}$ Irene de L. Noronha

Laboratório de Nefrologia Celular, Genética e Molecular

Faculdade de Medicina - Universidade de São Paulo

Av. Dr. Arnaldo, 455 - $4^{0}$ andar - S/4304 - São Paulo / SP - CEP: 01246-903 - Brasil

Tel.: (11) 30618401 - Fax: (11) 30618361

E-mail: irenenor@usp.br

\section{IMUNOSSUPRESSÃO E PROTEINÚRIA}

A proteinúria é um importante marcador de doença renal crônica, sendo reconhecida como fator de pior prognóstico, tanto na nefropatia diabética como em portadores de glomerulopatias e hipertensão arterial. ${ }^{1}$ Em pacientes submetidos a transplante renal, o aparecimento de proteinúria também tem importante significado clínico. ${ }^{2}$

Iniciamos esta revisão apresentando as principais teorias envolvidas no desencadeamento da proteinúria e, a seguir, comentaremos especificamente a proteinúria pós-transplante e sua relação com o uso de imunossupressores.

\section{Mecanismos envolvidos na proteinúria}

As bases anatômicas e funcionais da permeabilidade glomerular a macromoléculas têm sido propostas ao longo dos últimos 60 anos. Experimentos iniciais demonstraram que proteínas com baixo peso molecular eram excretadas na urina, enquanto proteínas com peso molecular maior eram retidas nos glomérulos. A partir destes achados na década de 50, foi desenvolvida a teoria dos poros, segundo a qual o fator limitante para a passagem de macromoléculas através dos poros nas paredes dos capilares seria seu tamanho. ${ }^{3}$ Assim, os solventes passariam livremente, enquanto que os solutos seriam retidos de acordo com suas dimensões. Algum tempo depois, foi descrita a teoria das cargas elétricas, que demonstrou que a carga elétrica das moléculas e não apenas seu 
Figura 1. Figura ilustrativa da anatomia molecular dos podócitos representando as principais proteínas que fazem parte da estrutura tipo "zíper" da fenda diafragmática (Adaptado de Zoysa \& Topham, $2005{ }^{59}$ e Kerjaschki, $2001^{60}$ )

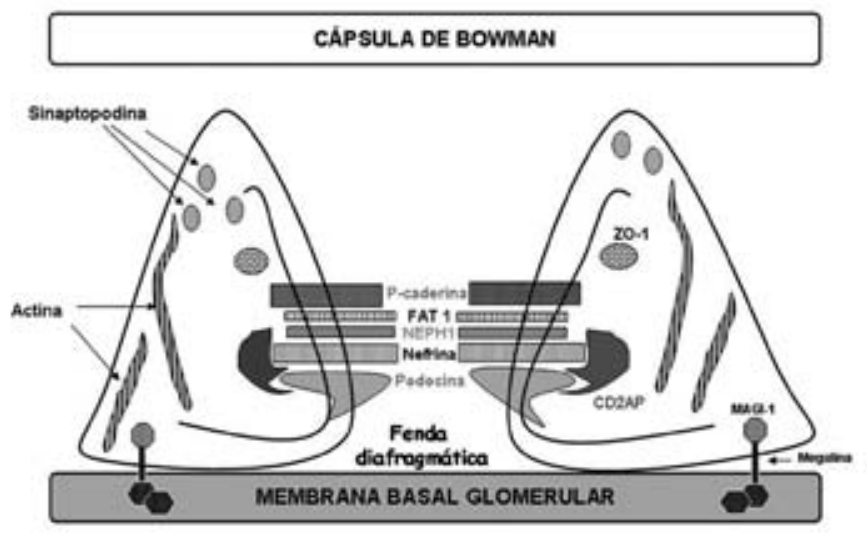

tamanho determinaria sua retenção ou não pela barreira de filtração glomerular. ${ }^{4,5} \mathrm{~A}$ partir de estudos com dextran com diferentes características de carga elétrica, observou-se um perfil diferente do clearance fracional destas moléculas. A administração de dextran sulfato (molécula com carga elétrica negativa) se associou a uma diminuição significativa da excreção urinária, quando comparada com a molécula de dextran neutro. Por outro lado, moléculas de dextran ligadas a radicais positivos promoveram aumento da excreção urinária.

Atualmente, a teoria mais abordada associa o aparecimento da proteinúria com alterações da fenda diafragmática dos podócitos. ${ }^{6}$ A fenda diafragmática possui uma estrutura tridimensional de proteínas interconectadas formando uma estrutura tipo "zíper" (Figura 1). Diversos estudos demonstraram que a ocorrência de alterações nessa estrutura está diretamente envolvida no desenvolvimento da proteinúria. ${ }^{7-9}$ Entretanto, o mecanismo que levaria a tais alterações ainda não foi esclarecido.

Várias moléculas que compõem a fenda diafragmática já foram identificadas (Figura 1). Dentre estas, destacam-se a nefrina, NEPH1, ZO1, FAT1, podocina, CD2AP, sinaptopodina, megalina, MAGI-1 e P-caderina. Essas proteínas estão intimamente ligadas aos podócitos e à fenda diafragmática, sendo responsáveis pela integridade da estrutura podocitária, e, conseqüentemente, pelo funcionamento adequado da barreira de filtração, impedindo assim a perda de proteínas urinárias. A deficiência na expressão dessas moléculas resulta em proteinúria. ${ }^{10-11}$

\section{Proteinúria pós-transplante}

Nos últimos anos, tem-se dado destaque especial à presença da proteinúria no pós-transplante como fator prognóstico de função do enxerto renal. Roodnat e cols. demonstraram que proteinúria maior que $1 \mathrm{~g} /$ dia aumenta tanto o risco de perda do enxerto renal como a mortalidade dos pacientes. ${ }^{12} \mathrm{O}$ nível de proteinúria correlaciona-se diretamente com o risco pós-transplante, sendo que quanto maior a perda de proteínas urinárias, pior sobrevida do rim transplantado e maior mortalidade cardiovascular. ${ }^{13,14}$ Não apenas a proteinúria, mas também a microalbuminúria é considerada fator de pior prognóstico da função do enxerto renal e sobrevida do receptor (tabela 1). ${ }^{15}$
A proteinúria em pacientes transplantados renais pode ter diversas causas, tais como recidiva da doença de base, glomerulopatia de novo, nefropatia crônica do enxerto, glomerulopatia do transplante, entre outras. A glomeruloesclerose segmentar e focal se caracteriza por episódios de recidivas mais precoces póstransplante, provavelmente em decorrência de fatores circulantes capazes de alterar a permeabilidade da barreira de filtração. No entanto, até o momento, esses possíveis “fatores humorais” ainda não foram identificados. A Tabela 2 apresenta a taxa de recidiva das glomerulopatias de maior importância clínica, com números variáveis dependendo da indicação da biópsia, número de pacientes de cada amostra e tempo de seguimento.

A glomerulopatia de novo, situação clinicamente menos freqüente, é diagnosticada quando após o transplante ocorre o aparecimento de uma glomerulonefrite em pacientes cuja doença de base não tenha sido glomerulopatia. A nefropatia crônica do enxerto também pode se manifestar com graus variáveis de proteinúria, sendo esta, inclusive, um dos seus marcadores. Finalmente, a glomerulopatia do transplante caracteriza-se por redução gradativa da função do enxerto renal, geralmente acompanhada de hipertensão arterial e proteinúria, sendo uma condição clínica de pior prognóstico. ${ }^{16}$ A partir da classificação de Banff publicada em 2007, a presença de depósitos de C4d passou a ser considerada importante para o diagnóstico de glomerulopatia do transplante. ${ }^{17}$

\section{Proteinúria e imunossupressão}

O uso de imunossupressores no tratamento de nefropatias proteinúricas já está bem estabelecido, como é o caso dos corticosteróides utilizados tanto no tratamento das lesões mínimas como em outras glomerulopatias primárias. ${ }^{18,19}$

Tabela 1. Proteinúria pós-transplante: prevalência e risco relativo de perda do enxerto renal

\begin{tabular}{lcc}
\hline Estudos & Proteinúria pós-Tx & Perda do enxerto \\
\hline Roodnat et al., 2001 ${ }^{12}$ & $31 \%$ & 2,03 \\
Fernandez-Fresnedo et al., $2004^{13}$ & $15,3 \%$ & 3,46 \\
Halimi et al., 2007 ${ }^{15}$ & $10,2 \%$ & $16,41^{*}$ \\
Kang et al., 2009 ${ }^{14}$ & $7,8 \%$ & 8,75 \\
\hline
\end{tabular}

* Odds Ratio macroalbuminúria x microalbuminúria

Tabela 2. Risco de recidiva das glomerulopatias no pós-transplante renal baseado em Choy et al., $2006^{47}$

\begin{tabular}{lcc}
\hline Glomerulopatia & Risco de recidiva & Risco de perda do enxerto \\
\hline Glomeruloesclerose segmentar e focal & $20-50 \%$ & $13-20 \%$ \\
GN Membrano-Proliferativa tipo I & $20-25 \%$ & $15 \%$ \\
GN Membrano-Proliferativa tipo II & $80-100 \%$ & $10-30 \%$ \\
Nefropatia da IGA & $13-46 \%$ & $2-16 \%$ \\
GN Membranosa & $10-30 \%$ & $10-15 \%$ \\
\hline
\end{tabular}


Tabela 3. Prevalência de proteinúria em pacientes transplantados recebendo inibidores do sinal de proliferação

\begin{tabular}{|c|c|c|c|}
\hline Estudos & PSIs & Protocolo & $\begin{array}{l}\text { Proteinúria * } \\
\text { (\% ou g/24h) }\end{array}$ \\
\hline Bumbea et al., $2005^{48}$ & SRL & conversão & $33 \%$ \\
\hline Letavernier et al., $2005^{29}$ & SRL & conversão & $46 \%$ \\
\hline Ruiz et al., $2005^{49}$ & SRL & conversão & $25 \%$ \\
\hline \multirow[t]{2}{*}{ Franco et al., $2007^{31}$} & SRL & conversão & $23 \%$ \\
\hline & & de novo & $13 \%$ \\
\hline Abramowicz et al., $2008^{50}$ & SRL & conversão & $18 \%$ \\
\hline Ducloux et al., $2002^{51}$ & SRL & conversão & de 0,8 para 1,8 \\
\hline Dervaux et al., $2005^{52}$ & SRL & conversão & de 0,5 para 1,8 \\
\hline Saurina et al., $2006^{53}$ & SRL & conversão & de 0,1 para 1,1 \\
\hline Boratynska et al., $2006^{54}$ & SRL & conversão & de 0,5 para 1,8 \\
\hline van der Akker et al., $2006^{55}$ & SRL & conversão & $1,8^{*}$ \\
\hline \multirow[t]{2}{*}{ Letavernier et al., $2007^{34}$} & SRL & conversão & de 0,3 para 2,5 \\
\hline & & de novo & entre 7 e 13 \\
\hline Sanchez-Fructuoso et al., $2007{ }^{56}$ & EVL & conversão & aumento $(>0,8)$ \\
\hline \multirow[t]{2}{*}{ Ruiz et al., $2007^{57}$} & EVL & conversão & de 0,4 para 0,6 \\
\hline & & de novo & de 0,8 para 0,4 \\
\hline Nowacka-Cieciura et al., 200958 & EVL & conversão & aumento \\
\hline
\end{tabular}

PSIs: inibidores do sinal de proliferação; SRL: sirolimus; EVL: everolimus

* pacientes apresentavam proteinúria antes da conversão

Mais recentemente, os inibidores da calcineurina (iCN) passaram a representar uma alternativa terapêutica nessa situação clínica, devido a seu efeito antiproteinúrico. Diversos estudos demonstram que o uso de ciclosporina está plenamente estabelecido para tratamento de glomerulopatias corticorresistentes, embora estudos recentes também comprovem a eficácia do tacrolimo. ${ }^{20-23}$ Em pacientes transplantados renais, é possível que o uso de iCN proporcione efeito terapêutico indireto, limitando a excreção de proteínas urinárias.

Estudos experimentais com micofenolato mofetil (MMF) em modelos de nefropatia crônica demonstraram diminuição da proteinúria e renoproteção. ${ }^{24-26}$ Atualmente, o MMF é utilizado clinicamente em alguns casos de glomerulopatias, nefrite lúpica e vasculite. ${ }^{27}$

Os inibidores do sinal de proliferação (proliferation signal inhibitors - PSIs), sirolimus e everolimus, são potentes drogas imunossupressoras utilizadas em transplante de órgãos, que bloqueiam a atividade do alvo da rapamicina em mamíferos (mammalian target of rapamycin - mTOR). Porém, seu uso em casos de transplante de novo ou em casos de conversão tem sido correlacionado com o aparecimento ou aumento da proteinúria (tabela 3). ${ }^{28-30}$
O aparecimento de proteinúria em pacientes transplantados em uso de PSIs descrito na literatura varia de 13\% a 45\% (tabela 3) e se apresenta de forma variável desde uma proteinúria discreta, podendo alcançar níveis nefróticos. ${ }^{31}$ No entanto, um aspecto clínico interessante é o fato de ocorrer reversão da proteinúria com a suspensão dos PSIs, principalmente quando realizada de forma precoce, sugerindo um efeito direto desta droga na indução da proteinúria. ${ }^{32,33}$ Quanto aos achados histológicos, a lesão mais frequentemente encontrada é a glomeruloesclerose segmentar e focal. . $^{31,34}$

Os mecanismos envolvidos na proteinúria pós-uso de PSIs ainda não foram elucidados. Uma possibilidade para o desenvolvimento da proteinúria seria o possível efeito dos PSIs sobre os podócitos. Podócitos humanos cultivados com inibidores da mTOR, mais especificamente a rapamicina, apresentaram diminuição da expressão de nefrina in vitro, confirmando que mTOR regula a expressão de proteínas da fenda diafragmática e do citoesqueleto dos podócitos. ${ }^{35}$

Um dos mediadores mais importantes responsáveis pela integridade dos podócitos é o fator de crescimento vascular endotelial (VEGF). ${ }^{36}$ Experimentalmente, o bloqueio de VEGF promove o aumento da excreção urinária de proteínas. ${ }^{37}$ Além disso, outros relatos descrevem que esse bloqueio pode estar associado à diminuição da expressão de nefrina. ${ }^{38}$ É importante ressaltar que estudos recentes demonstraram que o uso de PSIs promove não apenas o retardo do crescimento de alguns tumores, mas também diminui a incidência de câncer e reduz a progressão de metástases já existentes. ${ }^{39-41}$ Esses efeitos, aparentemente, são secundários à diminuição da produção do VEGF induzido pelos PSIs. ${ }^{42,43}$ Considerando que os podócitos e a fenda diafragmática são estruturas cruciais para a integridade da barreira de filtração e que os PSIs diminuem a síntese de VEGF, é possível que o desenvolvimento de proteinúria durante o uso de PSIs esteja relacionado primariamente à menor expressão de VEGF.

Visando o controle da proteinúria, uma estratégia clínica é o uso de bloqueadores do sistema renina-angiotensina, com conseqüente diminuição da pressão intraglomerular, já utilizado em alguns centros. ${ }^{44}$ Estudos clínicos, multicêntricos, randomizados estão atualmente em andamento para analisar criteriosamente o possível efeito benéfico de inibidores de enzima de conversão e/ ou bloqueadores dos receptores de angiotensina nesta situação. Além disso, a utilização de PSIs deve ser evitada em transplantados renais com clearance de creatinina menor que $40 \mathrm{ml} / \mathrm{min}^{45} \mathrm{ou} \mathrm{com}$ proteinúria maior que $0,8 \mathrm{~g} / \mathrm{dia},{ }^{46}$ critérios estes definidos a partir de trabalhos que analisaram fatores preditivos de pior prognóstico na função e sobrevida do enxerto.

Em resumo, existe maior prevalência de proteinúria associada ao uso de PSIs, cujo mecanismo ainda não foi totalmente elucidado. Novas pesquisas envolvendo os PSIs devem ser realizadas, para que seja possível melhor compreensão dessa manifestação clínica, assim como sua prevenção e tratamento, proporcionando maior sobrevida do paciente transplantado e do próprio enxerto renal. 


\section{ABSTRACT}

Proteinuria is an important marker for renal injury, not only in chronic kidney disease but also in kidney transplantation. In kidney transplant patients, proteinuria may have different causes, such as recurrence of the underlying disease, chronic allograft nephropathy, transplant glomerulopathy and de novo glomerulopathy. Immunosuppressive drugs used in transplantation interfere in the urinary excretion of proteins. Calcineurin inhibitors have important antiproteinuric effect, and they are being used to treat primary glomerulopathy. Antiproteinuric effect was also described using micophenolate. However, more recent studies have described the appearance and/or increase of proteinuria related to the use of proliferative signal inhibitors (PSIs). There are clinical evidences that proteinuria responds to the use of angiotensin-converting enzyme inhibitors or angiotensin-receptor blockers. In this context, the study of the effect of PSIs on the molecules of the podocyte slit diaphragm as nephrin, podocin and synaptopodin (among others), and also on vascular endothelial growth factor (VEGF) is relevant. This review covers the above mentioned aspects.

Keywords: Kidney Transplantation, Proteinuria, Immunosuppressive, Podocytes; Vascular endothelial growth factor.

\section{REFERÊNCIAS}

1. Taal MW, Brenner BM. Renal risk scores: progress and prospects. Kidney Int. 2008;73:1216-9.

2. Reichel H, Zeier M, Ritz E. Proteinuria after renal transplantation: pathogenesis and management. Nephrol Dial Transplant. 2004;19:301-5.

3. Pappenheimer JR, Renkin EM, Borrero LM. Filtration, diffusion and molecular sieving through peripheral capillary membranes, a contribution to the pore theory of capillary permeability. Am J Physiol. 1951;167:13-46.

4. Brenner BM, Hostetter TH, Humes HD. Glomerular permselectivity: barrier function based on discrimination of molecular size and charge. Am J Physiol. 1978;234:F455-60.

5. Ohlson M, Sorensson J, Haraldsson B. Glomerular size and charge selectivity in the rats as revealed by FITC-ficoll and albumin. Am J Physiol Renal Physiol. 2000;279:F84-91.

6. Akhtar M, Al Mana H. Molecular basis of proteinuria. Adv Anat Pathol. 2004;11:304-9.

7. Patrakka J, Kestila M, Wartiovaara J, Ruotsalainen V, Tissari P, Lenkkeri U, et al Congenital nephrotic syndrome (NPHS1): features resulting from different mutations in finnish patients. Kidney Int. 2000;58:972-80.

8. Doublier S, Ruotsalainen V, Salvidio G, Lupia E, Biancone L, Conaldi PG, et al. Nephrin redistribution on podocytes is a potential mechanism for proteinuria in patients with primary acquired nephrotic syndrome. Am J Pathol. 2001;158:1723-31.

9. Lee YK, Kwon T, Kim DJ, Huh W, Kim Y, Oh H, et al. Ultrastructural study on nephrin expression in experimental puromicyn aminonucleoside nephrosis. Nephrol Dial Transplant. 2004;19:2981-86.

10. Shih N, Li J, Cotran R, Mundel P, Miner JH, Shaw AS. CD2AP localizes to the slit diaphragm and binds to nephrin via a novel c-terminal domain. Am J Pathol. 2001;159:2303-08.

11. Kim BS, Park HC, Kang SW, Choi KH, Ha SK, Han DS, et al. Impact of cyclosporine on podocyte $\mathrm{ZO}-1$ expression in puromycin aminonucleoside nephrosis rats. Yonsei Med J. 2005;46:141-48.

12. Roodnat JI, Mulder PGH, Rischen-Vos J, Van Riemsdijk IC, Van Gelder T, Zietse $\mathrm{R}$, et al. Proteinuria after renal transplantation affects not only graft survival but also patient survival. Transplantation. 2001;72:438-44.

13. Fernandez-Frenesdo G, Plaza JJ, Sanchez-Plumed J, Sanz-Guajardo A, PalomaFontanet R, Arias M. Proteinuria: a new marker of long-term graft and patient survival in kidney transplantation. Nephrol Dial Transplant. 2004;19:47-51.

14. Kang NR, Lee JE, Huh W, Kim SJ, Kim Y, Kim DJ, et al. Minimal proteinuria one year after transplant is a risk factor for graft survival in kidney transplantation. J Korean Med Sci. 2009;24:5129-34.
15. Halimi JM, Buchler M, Al-Najjar A, Laouad I, Chatelet V, Marliere JF, et al. Urinary albumin excretion and the risk of graft loss and death in proteinuric and non-proteinuric renal transplant patients. Am J Transplant. 2007;7:618-25.

16. Akalin E, Dikman S, Murphy B, Bromberg JS, Hancock WW. Glomerular infiltration by CXCR3+ ICOS+ activated T cells in chronic allograft nephropathy with transplant glomerulopathy. Am J Transplant. 2003;3:1116-20.

17. Solez K, Colvin RB, Racusen LC, Haas M, Sis B, Mengel M, et al. Banff 07 classification of renal allograft pathology: updates and future directions. Am J Transplant. 2008;8:753-60.

18. Bargman JM. Management of minimal lesion glomerulonephritis: evidence-based recommendations. Kidney Int Suppl. 1999;70:S3-16.

19. Chun MJ, Korbet SM, Schwartz MM, Lewis EJ. Focal segmental glomerulosclerosis in nephrotic adults: presentation, prognosis, and response to therapy of the histologic variants. J Am Soc Nephrol. 2004;15:2169-77.

20. Ponticelli C, Rizzoni G, Edefonti A, Altieri P, Rivolta E, Rinaldi S, et al. A randomized trial of cyclosporine in steroid-resistant idiopathic nephrotic syndrome. Kidney Int. 1993;43:1377-84.

21. Cattran DC, Appel GB, Hebert LA, Hunsicker LG, Pohl MA, Hoy WE, et al. A randomized trial of cyclosporine in patients with steroid-resistant focal segmental glomerulosclerosis. North America Nephrotic Syndrome Study Group. Kidney Int. 1999;56:2220-6.

22. Segarra A, Vila J, Pou L, Majo J, Arbos A, Quiles T, et al. Combined therapy of tacrolimus and corticosteroids in cyclosporin-resistant or -dependent idiopathic focal glomerulosclerosis: a preliminary uncontrolled study with prospective follow-up. Nephrol Dial Transplant. 2002;17:655-62.

23. Duncan N, Dhaygude A, Owen J, Cairns TD, Griffith M, Mclean AG, et al. Treatment of focal and segmental glomerulosclerosis in adults with tacrolimus monotherapy. Nephrol Dial Transplant. 2004;19:3062-7.

24. Fujihara CK, Malheiros DM, Zatz R, Noronha IL. Mycophenolate mofetil attenuates renal injury in the rat remnant kidney. Kidney Int. 1998;54:1510-9.

25. Utimura R, Fujihara CK, Mattar AL, Malheiros DM, Noronha IL, Zatz R. Mycophenolate mofetil prevents the development of glomerular injury in experimental diabetes. Kidney Int. 2003;63:209-16.

26. Ryuzo M, Soares V. Effect of mycophenolate mofetil on the progression of adriamycin nephropathy. Ren Fail. 2001;23:611-9.

27. Appel AS, Appel GB. An update on the use of mycophenolate mofetil in lupus nephritis and other primary glomerular diseases. Nature Rev Nephrol. 2009;5:132-42.

28. Diekmann F, Budde K, Oppenheimer F, Fritsche L, Neumayer HH, Campistol JM. Predictors of success in conversion from calcineurin inhibitor to sirolimus in chronic allograft dysfunction. Am J Transplant. 2004;4:1869-75. 
29. Letavernier E, Peraldi MN, Pariente A, Morelon E, Legendre C. Proteinuria following a switch from calcineurin inhibitors to sirolimus. Transplantation. 2005;80:1198-203.

30. Morales JM, Grinyo JM, Campistol JM, Garcia-Martinez J, Arias M, Paul J, et al. Improved renal function, with similar proteinuria, after two years of early tacrolimus withdrawal from a regimem of sirolimus plus tacrolimus. Transplantation. 2008;86:620-2.

31. Franco AFV, Martini Filho D, Abensur H, Noronha IL. Proteinuria in transplant patients associated to sirolimus. Transplantation Proc. 2007;39:449-52.

32. Dittrich E, Schmaldienst S, Soleiman A, Horl WH, Pohanka E. Rapamycin-associated post-transplantation glomerulonephritis and its remission after reintroduction of calcineurin-inhibitor therapy. Transplant Int. 2004;17:215-20.

33. Senior PA, Paty BW, Cockfield SM, Ryan EA, Shapiro AM. Proteinuria developing after clinical islet transplantation resolves with sirolimus withdrawal and increases tacrolimus dosing. Am J Tranplant. 2005;5:2318-23.

34. Letavernier E, Bruneval P, Mandet C, Van Huyen JP, Péraldi MN, Helal I, et al. High sirolimus levels may induce focal segmental glomerulosclerosis de novo. Clin J Am Soc Nephrol. 2007;2:326-33.

35. Vollenbröker B, George B, Wolfgart M, Saleem MA, Pavenstädt H, Weide T. mTOR regulates expression of slit diaphragm proteins and cytoskeleton structure in podocytes. Am J Physiol Renal Physiol. 2009;296:F418-26.

36. Foster RR, SatchelL SC, Seckley J, Emmett MS, Joory K, Xing CY, et al. VEGF-C promotes survival in podocytes. Am J Physiol Renal Physiol. 2006;291:196-07.

37. Sugimoto H, Hamano Y, Charytan D, Cosgrove D, Kieran M, Sudhakar A, et al. Neutralization of circulating vascular endothelial growth factor (VEGF) by antiVEGF antibodies and soluble VEGF receptor 1 (sFlt-1) induces proteinuria. J Biol Chem. 2003;278:12605-8

38. Hara A, Wada T, Furuichi K, Sakai N, Kawachi H, Shimizu F, et al. Blockade of VEGF accelerates proteinuria, via decrease in nephrin expression in rat crescentic glomerulonephritis. Kidney Int. 2006;69:1986-95.

39. Dasanu CA, Clark BA 3rd, Alexandrescu DT. mTOR-blocking agents in advanced renal cancer: an emerging therapeutic option. Expert Opin Investig Drugs. 2009;18:175-87.

40. Böhm A, Aichberger KJ, Mayerhofer M, Herrmann H, Florian S, Krauth MT, et al. Targeting of mTOR is associated with decreased growth and decreased VEGF expression in acute myeloid leukaemia cells. Eur J Clin Invest. 2009;39:395-05.

41. Konings IR, Verweij J, Wiemer EA, Sleijfer S. The applicability of mTOR inhibition in solid tumors. Curr Cancer Drug Targets. 2009;9:439-50.

42. Thaunat $\mathrm{O}$, Morelon E. Cancer and immunosuppression: pro and antitumoral effects of immunosuppressive drugs. Nephrol. Ther. 2005;1:23-30.

43. Stallone G, Infante B, Grandaliano G, Schena FP, Gesualdo L. Kaposi's sarcoma and mTOR: a crossroad between viral infection neoangiogenesis and immunosuppression. Transplant Int. 2008;21:825-32

44. Cruzado JM, Rico J, Grinyó JM. The renin angiotensin system blockade in kidney transplantation: pros and cons. Transpl Int. 2008;21:304-13.

45. Stallone G, Infante B, Schena A, Battaglia M, Ditonno P, Loverre A, et al. Rapamycin for treatment of chronic allograft nephropathy in renal transplant patients. J Am Soc Nephrol. 2005;16:3755-62.
46. Diekmann F, Budde K, Oppenheimer F, Fritsche L, Neumayer HH, Campistol JM. Predictors of success in conversion from calcineurin inhibitor to sirolimus in chronic allograft dysfunction. Am J Transplant. 2004;4:1869-75.

47. Choy BY, Chan TM, Lai KN. Recurrent glomerulonephritis after kidney transplantation. Am J Transplant. 2006;6:2535-42.

48. Bumbea V, Kamar N, Ribes D, Esposito L, Modesto A, Guitard J, et al. Long-term results in renal transplant patients with allograft dysfunction after switching from calcineurin to sirolimus. Nephrol Dial Transplant. 2005;20:2517-23.

49. Ruiz JC, Diekmann F, Campistol JM, Sanchez-Fructuoso A, Rivera C, Oliver J, et al. Evolution of proteinuria after conversion from calcineurin inhibitors (CNI) to sirolimus (SRL) in renal transplant patients: a multicenter study. Transplant Proc. 2005;37:3833-5.

50. Abramowicz D, Hadaya K, Hazzan M, Broeders N, Hoang AD, Ghisdal L, et al. Conversion to sirolimus for chronic renal allograft dysfunction: risk factors for graft loss and severe side effects. Nephrol Dial Transplant. 2008;23:3727-9.

51. Ducloux D, Motte G, Billerey C, Bresson-Vautrin C, Vautrin P, Rebibou JM, et al. Cyclosporin withdrawal with concomitant conversion from azathioprine to mycophenolate mofetil in renal transplant recipients with chronic allograft nephropathy: a 2-year follow-up. Transplant Int. 2002;15:387-92.

52. Dervaux T, Caillard S, Meyer C, Ellero B, Woehl-Jaegle ML, Hannedouche T, et al. Is sirolimus responsible for proteinuria? Transplant Proc. 2005;37:2828-9.

53. Saurina A, Campistol JM, Piera C, Diekmann F, Campos B, Campos N, et al. Conversion from calcineurin inhibitors to sirolimus in chronic allograft dysfunction: changes in glomerular haemodynamics and proteinuria. Nephrol Dial Transplant. 2006;21:488-93.

54. Boratynska M, Banasik M, Watorek E, Falkiewicz K, Patrzalek D, Szyber P, et al. Conversion to sirolimus from cyclosporine may induce nephrotic proteinuria and progressive deterioration of renal function in chronic allograft nephropathy patients. Transplant Proc. 2006;38:101-04.

55. Van der Akker JM, Wetzels JFM, Hoitsma AJ. Proteinuria following conversion from azathioprine to sirolimus in renal transplant recipients. Kidney Int. 2006;70:1355-7.

56. Sanchez Fructuoso A, Ruiz San Millán JC, Calvo N, Rodrigo E, Moreno MA, Cotorruelo J, et al. Evaluation of the efficacy and safety of the conversion from a calcineurin inhibitor to an everolimus-based everolimus-based therapy in maintenance renal transplant patients. Transplantation Proc. 2007;39:2148-50.

57. Ruiz JC, Sánchez A, Rengel M, Beneyto I, Plaza JJ, Zárraga S, et al. Use of the new proliferation signal inhibitor everolimus in renal transplant patients in Spain: preliminary results of the everodata registry. Transplant Proc. 2007;39:2157-9.

58. Nowacka-Cieciura E, Perkowska-Ptasińska A, Sulikowska-rowińska A, Cieciura T, Wazna E, Durlik M. Late conversion to everolimus complicated with necrotizing glomerulonephritis in a renal allograft recipient: case report. Transplantation Proc. 2009; $41: 441-5$.

59. Zoysa JR, Topham PS. Podocyte biology in human disease. Nephrology. 2005; $10: 362-7$.

60. Kerjaschki D. Caught flat-footed: podocyte damage and the molecular bases of focal glomerulosclerosis. J Clin Invest. 2001;108:1583-7. 